prognosis for pelvic delivery is very good and improves as either diameter increases above the minimum. In my tables only seven cases of this degree are included, in which some trouble occurred; two of these were markedly rachitic, and in four others the foetus was considerably above the average size.

Medium Degree.-The medium degree includes all cases with the conjugate reduced to $9 \mathrm{~cm}$., but not less than $8 \mathrm{~cm}$., and a transverse reduced below normal but not less than $10.75 \mathrm{~cm}$. These measurements are represented by an external conjugate of $18 \mathrm{~cm}$. but not less than $16 \frac{1}{2} \mathrm{~cm}$., and an intercristal reduced to $25 \frac{1}{2} \mathrm{~cm}$. When the conjugate is reduced to $9 \mathrm{~cm}$. it precludes the passage of an average biparietal diameter, but the average bitemporal diameter is about $8.25 \mathrm{~cm}$., and capable of slight reduction. The prognosis in this degree depends upon to what extent the bitemporal can be substituted for the biparietal, and this depends upon the transverse diameter. When the transverse is normal, the maximum exchange can occur, the pelvis is simple flat in type, and the prognosis is good even with the conjugate reduced to $8 \mathrm{~cm}$. On the other hand, with the transverse reduced to $10.75 \mathrm{~cm}$. no change from the biparietal can occur, and its passage depends upon moulding, which has reached its limit of safety at $9 \mathrm{~cm}$. The pelvis is transversely contracted in type, and the prognosis, although good, is not better than the simple flat with an $8 \mathrm{~cm}$. conjugate. As both diameters are gradually reduced the prognosis gradually gets worse. Of my cases 157 come into this degree, and 23, or 14.6 per cent., had difficulty greater than low forceps or were premature.

Major Degree.-The major degree of contraction is when the conjugate is reduced to $8 \mathrm{~cm}$. No improvement is obtained even with a normal transverse, as the space is not compatible with the average bitemporal diameter. The prognosis is bad for pelvic delivery. Twenty-six cases come into this degree, and 9, or 34.6 per cent., were difficult.

In all three degrees of contraction the prognosis is improved by a reduction of the foetal head below the normal average, while increase in the size of the head makes the prognosis worse.

\section{CARCINOMA OF THE COLON.*}

BY

W. H. C. ROMANIS, M.A., M.B., M.Сh.Cantab., F.R.C.S.ENG.,

ASSISTANT SURGEON, ST. THOMAS'S HOSPITAL; SURGEON, CITY OF LONDON HOSPITAL FOR DISEASES OF THE CHEST.

('Arcinoma of the colon is a disease which is both common and insidious, which is absolutely certain to end the patent's life if not properly treated, but which may take several year's to do so; it is probably one of the most favourable forms of malignant disease which is found anywhere in the body, for it is slow-growing, remains long confined to the bowel wall, does not spread rapidly to the neighbouring glands, and only tends to form metastases rather late in its course. Unfortunately its symptoms are insidious and equivocal, and it is owing to this that its treatment is often delayed, so that the results are at present sometimes not so satisfactory as they might be. It is well known that one of the main symptoms of malignant disease of the large bowel is obstruction, more or less acute, and perhaps it is fortunate that in many cases this onset of obstruction occurs early in the disease and calls attention to the need of operation, though it would really be greatly preferable for operation to be performed if possible before the obstruction sets in.

In this paper I intend to deal only with cases of carcinoma of the true colon, and do not include the rectum, for two reasons: first, because the diagnosis of the rectal cases is very much more easy and straightforward; and secondly, because, the rectum being an extraperitoneal organ, the operative treatment of carcinoma, when it occurs there, is rather different.

Malignant disease of the large bowel is, of course, most

* Extracts from a paper read to the Shrewsbury Division of the British Medical Association. commonly seen in the pelvic colon and caecum, but we meet with it not infrequently in the splenic flexure and the transverse and descending colons, while it is least commonly found at the hepatic flexure. Microscopically it is usually of a true columnar type, but to the naked eve it appears in three very distinct forms, and I wish to lay especial stress on these three naked-eye types, for they have an important influence in both diagnosis and treat. ment. The first type, or Type $A$, is the annular or ring carcinoma, which is the commonest form and gives the well known appearance of a string tied round the bowel; this is more common on the left side of the colon than the right, and it naturally tends to produce obstructive symptoms earlier than do the other forms. The second form, or Type $B$, is the proliferating or fungating type, which grows into the bowel lumen and forms a large tumour and a palpable swelling. This is commoner in the right side of the colon than the left, and is of comparatively slow growth. The third type, or Type $\mathbf{C}$, is the hard, craggy ulcer, which grows down into the bowel wall and infiltrates it widely. This is, fortunately, rare in the colon: though common in the rectum, and I say fortunately because it is the most malignant of the three types, most prone to cause metastases, and also to give rise to the fewest and least noticeable symptoms. The relative malignancy of these types is also of importance in the question of operative technique, as we shall see later, for it may greatly influence the extent and scope of the operation to be performed in any individual case: Type $C$ is the most malignant, Type $B$ the least malignant, and Type $A$ is of an intermediate degree of malignancy.

Course and Diagnosis.

In the diagnosis of this condition there are several features on which I should like to lay especial stress. Most of these cases which a surgeon sees are either in a state of acute obstruction, or else come complaining of constipation and chronic obstruction, or of a tumour in the abdomen.

Now all these onditions must be regarded as late developments in the case, comparable in a way to the development of peritonitis in a case of acute appendicitis, or of coma in a case of cerebral tumour. Earlier diagnosis is admittedly difficult and may even be speculative, but it is most important that it should be made if possible, and there are usually certain symptoms which may give rise to a sufficiently strong suspicion to justify an exploratory operation. These are, in the first place, colicky pains and uncomfortable sensations after meals, diffused more or less through the abdomen, and often accompanied by loud borborygmi and gurglings. Sometimes a patient will state that these borborygmi and pains seem to pass up towards some particular spot in the abdomen and then disappear, and he will perhaps suggest that it feels as if there were a blockage at this point well before any really definite symptoms of obstruction are present. Secondly, there is nearly always a history of a certain amount of irregularity in the action of the intestines, either diarrhoea or constipation; and the right and left hand sides of the colon appear to behave very differently in this respect, for whereas in the case of a growth in the left side of the colon there is nearly always more or less marked constipation, in the right side of the colon a growth very rarely causes constipation, but more frequently diarrhoea and loose motions. It is well known that a growth at the extreme end of the colon and in the rectum will cause prolonged diarrhoea, but this is really a spurious diarrhoea and not quite comparable with that due to a carcinoma in the caecum and ascending colon; it serves to call attention, however, to the fact that every case of diarrhoea in an adult which persists for more than ten days and does not promptly react to medical treatment urgently demands examination to see if there is a malignant growth, either in the lower end or the upper part of the colon. The constipation, which in other cases is so marked a symptom, often gives the clue to the whole condition, and the sudden commencement of difficulty in getting the bowels to act in a middle-aged patient who has previously been perfectly regular in this respect is a most suspicious symptom. 
The difference in the type of intestinal irregularity which is produced by a carcinoma in the right-hand or lefthand side of the colon-namely, diarrhoea in the first case and constipation in the latter-is probably due to two facts :

(1) That the intestinal contents are far looser and more fluid in the caecum and ascending colon, and can therefore pass through any narrowed portion of the bowel more easily.

(2) That the type of carcinoma found at the two sides of the colon is usually different.

The commonest form on the left side is the ring carcinoma, and this, of course, soon narrows and obstructs the bowel, whereas in the right side of the colon we do not often see this variety, for the growth is more commonly of one of the other two types, and neither of these produces obstructive symptoms for some time. This being so it is usually those cases in which the growth is in the caecum or ascending colon which come to the surgeon complaining of a palpable swelling in the abdomen. Malignant disease of the colon is nearly always a slow-growing type of carcinoma, and the right side of the colon appears to give rise to growths which grow more slowly even than those in the left side; and it is not uncommon to find patients with growths of the caecum or ascending colon who have had symptoms for two or even three years and are yet toleraibly fit and well.

In addition to the symptoms of disordered intestinal function there are, as a rule, very definite changes to be found in the faeces: blood is probably always present, not usually in large quantities, and it may need chemical or microscopic examination for its detection; mucus also is usually present, and often this is commented upon by the patient. This presence of mucus in the stools is also of importance as affording some evidence as to the situation of the growth, for the nearer the carcinoma is to the lower end of the colon the greater the amount of mucus in the stools, while in the case of malignant disease of the caecuri or ascending colon mucus is not often detectable at all. Of this mucus a certain small amount comes from the ulcerated surface of the growth, but the bulk of it is probably secreted by the inflamed mucous membrane which is usually present above the growth. Anaemia is another symptom of importance which is often present, and though it is not so severe as a rule as in cases of carcinoma of the stomach, it is often a very marked feature. As soon as any slight tendency to obstruction sets in, often quite a long time before there are any signs of acute and absolute obstruction, there will be more or less distension of the abdomen, and wherever in the colon the growth is this distension will affect the caecum chiefly, and this distended caecum will be both visible and palpable.

Certain accessory methods of diagnosis are often of use: chemical examination of the faeces may demonstrate the presence of blood which cannot be detected with the naked eye, while $x$-ray examination with bismuth or barium is occasionally, but not often, of assistance. Bismuth or barium meals are, $I$ think, useless in this connexion, and if we are to gain any information in the case of disease of the colon the bismuth (or barium) must be administered by a high enema, and not by the mouth; in this case a " filling defect" or a constriction may occasionally be seen in the bowel lumen, but I do not personally place very much reliance upon the presence or absence of these signs. Rectal examination is, of course, of the greatest importance, for by its means not only may growths at the top of the rectum be felt, but at times carcinomata of the pelvic colon lying in the pelvis at the side of the rectum may be palpated through the rectal wall. Bimanual examination under an anaesthetic will sometimes detect a growth which is impalpable by any other means. Of almost equal importance is sigmoidoscopy, for by this means growths lying anywhere in the pelvic colon can be seen and an estimate of their size and operability made; moreover, if necessary, portions can be removed by this means for microscopy. We must remember that not only is the pelvic colon the commonest situation for these growths, but it is just in that portion of the bowel that a growth often cannot be felt, either by palpation or by rectal examination, and it is therefore of the greatest use to be able to find out the condition of the pelvic colon for certain by means of the sigmoidoscope.

Signs and Symptoms of Complete Obstruction.

I wish now to pass from the diagnosis of malignant disease of the colon in its early and pre-obstructive stage to a consideration of the signs and symptoms which it produces when it is giving rise to complete and more or less acute intestinal obstruction. This is, unfortunately, the stage in' which a great many cases are seen by the surgeon, and, owing to the fact that there are one or two ways in which obstruction from this cause differs from that due to other causes, the condition is at times allowed to progress for several days before surgical treatment is established. In a case of this kind there will usually be a history of symptoms pointing to a growth in the colon which have been present for some months, though this history will often take a considerable amount of extraction from the patient, and in fact in some cases it cannot be obtained. When the final obstruction supervenes, although the blockage is absolute and no motions or flatus may be passed at all, yet there is often an absence of some of the classical symptoms of acute intestinal obstruction, and the condition may not appear to be at all acute in nature. Pain and vomiting are often absent, at any rate for several days, and not infrequently the patient remains for a week or ten days without passing anything, without pain or vomiting, with a good pulse and a clean tongue; of ten the only symptom that points to the seriousness of the condition is the gradual and steadily progressive distension of the abdomen, which persists and after a few days may lead to a most enormous abdominal overdistension. This distension affects first of all the right flank and right iliac fossa, but soon involves the whole of the abdomen, which becomes uniformly blown up and tympanitic; inasmuch as there will soon be a certain amount of clear fluid exuding from the hyperdistended bowel into the peritoneum, shifting dullness may be present in the flanks, but we must not be led into regarding the whole condition as due to ascites because of this. The absence of pain and vomiting and the clean tongue, which are not infrequently seen, are probably due to two things: (1) we are here dealing with a pure obstruction or blockage of the bowel without any strangulation of its wall; (2) the fact that the obstruction is so low down that the whole of the upper part of the bowel, large and small, can act as a reservoir for the accumulated intestinal gases and products for a long time. The age of the patient, usually between 40 and 70 , the absence of any other discoverable cause for the obstruction, its gradual onset, and above all the presence of a palpable and grossly distended caecum and ascending colon, will usually render the diagnosis of the condition fairly certain.

\section{Treatment.}

In considering the treatment to be adopted in these cases, I think that we must regard the subject from two standpoints: first, what should be done in a case where obstruction, acute or subacute, is present; and secondly, what surgical methods are suitable in the early cases, in which there is no obvious obstruction. I would further subdivide the cases met with under four headings: those which are obstructed and where the growth is removable; the obstructed and irremovable; the non-obstructed and removable; and the non-obstructed and irremovable.

\section{Obstructed Cases.}

In the obstructed cases operation is, of course, a matter of more or less urgency, but a few hours' or even a day's delay is sometimes not of very great import, and I would urge that before any operation is undertaken for the relief of obstruction due to carcinoma of the colon the effect of one or two turpentine enemata should be tried. These are not, of course, likely to cure the obstruction, but they do occasionally relieve it, and if this is happily the case the operation to remove the growth can themobe done a few days later in a non-obstructed patient, and will prove far easier and safer and more satisfactory. In other cases the administration of these enemata 
will bring away a certain amount of flatus, and by so doing will lessen the distension and make the patient's condition better and the operation less troublesome. It must be remembered that operation in an obstructed case is primarily done to relieve the obstruction and not to remove the growth, and that when obstruction is present no radical operation of removal of the growth and anastomosis of the bowel must be attempted, for its risks are far too great. At the same time it is essential, when operating for obstruction due to carcinoma of the colon, to ascertain exactly where the growth is and whether it is removable or not, for the question of removing it later at a second operation, after the obstruction is relieved, is certain to arise. To do this it is best to make an incision below the umbilicus and slightly to the right of the mid-line and insert the hand; great care should be taken not to allow the distended coils of intestine to escape, for this will cause increased shock, and there may be difficulty in getting them back. The caecum should then be palpated to see if it is distended or empty, and remember that these very distended caeca are often easily burst by undue pressure. If the caecum is distended the obstruction is in the colon; if collapsed it is in the small bowel. In the case of a carcinoma of the colon the caecum will always be found to be distended, and the colon should then be examined, commencing in the pelvis, passing up the left side, across, and down the right side till the growth is felt. The growth should then quickly be examined, both by palpation and inspection if possible, and special attention paid to whether it is fixed or not, and whether there are secondary growths elsewhere, in lymphatic glands, the liver, the pelvis, etc.

Selection of Operation.

Having ascertained whether the growth is removable or not the obstruction must then be relieved, and there are three main ways of doing this: (1) By a colostomy or caecostomy above the growth. (2) By short-circuiting the growth by anastomosing the bowel above the growth to that below the growth. (3) By excising the growth, leaving the two ends of the bowel open and tring Paul's tubes into them. On no account must the growth be removed and the radical operation completed there and then in one stage by anastomosing the bowel ends, for the bowel wall, in its distended, sodden, and septic condition, will not hold the stitches well and disaster will result. There is little doubt that of these methods a caecostomy or colostomy is the safest and easiest, and if the growth is very low down in the colon it may be the only procedure to be done, for there will not be enough bowel below the growth for an anastomosis to be possible.

If, then, the growth is operable, so that the patient will probably wish to have it removed at a later operation in two or three weeks' time, a temporary caecostomy or temporary colostomy, done without too big a spur, so that it will close itself or can easily be closed later, is undoubtedly the best method of relieving the obstruction with a view to resection of the growth later. If, however, the growth is not operable and the question of removing it later does not arise, a colostomy, if done, will have to be permanent, and then we may hesitate rather as to whether it is not more pleasant for the patient to do a short-circuit rather than submit him to the unpleasantness of a permanent colostomy, even though the risk is slightly greater; and in these inoperable growths, unless the bowel wall is very distended and sodden, so that a short-circuit would carry a great deal of risk, I personally prefer, if possible, to do the anastomosis rather than a colostomy. In any case, if the growth is in the caecum and near the ileocaecal valve, there will not be room to do a caecostomy above it; and as an ileostomy is not a justifiable procedure, owing to the starvation and the digestion of the surrounding skin which it produces, a short-circuit between the end of the ileum and the colon below the growth is the only procedure to be done.

The third method, that of excising the growth during the operation for acute obstruction and tying Paul's tubes into both ends of the bowel, is variously ascribed to von Mikulicz and to Paul, but $I$ do not practise it, for it is an unnecessarily elaborate operation to undertake while the acute condition is present, and the necessarily distended condition of the bowel will interfere with the excision of the growth being as free and unhampered as it would be if performed later at a second operation.

The results of these operations for acute obstruction due to carcinoma of the colon are in some ways disappointing, for there is a very definite mortality attaching to them-somewhere in the neighbourhood of 25 per cent. Although at times the patient appears to be remarkably fit in his general condition, yet after even so simple a procedure as a straightforward colostomy sudden collapse and death sometimes occur, while it is not unusual for the colostomy to fail to act, and so for the obstruction not to be reliezed. In some instances this failure of the colostomy to act is due to the way in which it is done, for the bowel is hyperdistended and on the verge of becoming paralysed; if now the colostomy is performed by simply bringing the colon out through the abdominal wall and inserting a Paul's tube into the side of it, the constriction of the protruding gut by the abdominal muscles may be sufficient to prevent the semiparalysed bowel from expelling its contents. This can easily be prevented by using a prolongation of the Paul's tube which will run into the bowel for three or four inches, and which can be made by introducing a tightly fitting rubber tube into the Paul's tube so that the rubber stands out for two or three inches. This little prolongation will ensure that the way out for the contents of the colon is not unduly constricted, and since $I$ have-taken to using it there have been far fewer cases in which the colostomy has failed to act. Another wise proceeding is the introduction of one or two ounces of glycerin down the Paul's tube into the bowel as soon as the operation is finished.

We can now proceed to consider the question of the treatment of those cases which are not yet obstructed, and this, of course, largely depends upon the question of whether the growth is operable or not. It is in many cases exceedingly difficult to say whether any particular growth is going to be capable of removal or not until the abdomen is opened, though, of course, if the patient is very weak and debilitated, or has any other gross disease, or if secondary growths can be detected in the liver, glands, or elsewhere, we can feel certain that nothing in the way of removing the growth should be done. As a general rule a colonic growth which can be felt to be large -that is to say, which gives the impression of being bigger than a large orange-will very probably not be removable even though it is quite movable. For it will be found to have infiltrated extensively into the root of the mesentery and into the surrounding structures.

On opening the abdomen in one of these cases, then, the first thing to be done is to ascertain whether the growth is operable or not-if it is small and movable and there are no secondary growths it is, of course, capable of removal; a moderate amount of fixity by adhesions on to the posterior abdominal wall does not render it irremovable, nor does the presence of glands on the side of the bowel or in the mesentery close by. But if there is a big mass of glands at the root of the mesentery, or further up on the aorta, or in the hepatic fissure, or if there are nodules of secondary growth in the liver, the omentum, or the peritoneum, especially that of the pelvis, no attempt will be made to remove the primary growth. Should the growth for one or other of these reasons prove to be irremovable, there is nevertheless a step that can be taken which will relieve the symptoms, prevent obstruction, and prolong life considerably, and that is the performance of a shortcircuit above and below the growth. This is best done by means of a lateral anastomosis between the bowel above and that below the growth, but it is well to remember that in many cases the ileo-caecal valve is continent and prevents backward regurgitation from the caecum into the ileum: in this case, if the anastomosis is made between the ileum above the valve and the colon below the growth, the caecum will become distended with gas and secretions, which cannot pass down because of the growth, and cannot 
pass up because of the ileo-caecal valve, and not only will this cause pain and discomfort, but there is also considerable danger of the caecum bursting or perforating. This operation of short-circuiting an irremovable growth is well worth doing, for not only is malignant disease of the large bowel of slow growth, but when it is relieved from the irritation of faecal matter passing over it it will grow even more slowly. The way in which the growth destroys life early is, of course, by causing obstruction, and once this danger is removed by the short-circuit many patients live comfortably for a considerable time, often two or three year's; a colostomy, of course, achieves the same object, but it does so often with considerable permanent discomfort and distress to the patient.

Removable Growth Not Producing Obstruction.

We now come to our fourth and final class of case, in which there is no obstruction and the growth proves to be removable, and here our aim and object should be to excise the growth and reunite the ends of the bowel by some form of anastomosis straight away at one sitting. Even in this case, where the growth is removable, there are certain eminent authorities who prefer to make no attempt at all to remove the growth, but merely do the short-circuiting operation, described above, in all cases, for they say that the risk of performing a short-circuit is considerably less than that of removing the growth, and that if the growth is not removed, but a short-circuit is performed, the patient will probably live comfortably for two or three years; whereas even if the growth is removed he will probably suffer from a recurrence within two or three years. Theoretically these arguments are true enough, but personally I have no sympathy with the conclusions to which they lead, for by removing the growth we offer the patient a reasonable chance of a permanent cure, at a certain risk, and I think that most patients would prefer to face this rather than to have a certainty of death within two or three years and less immediate risk at the operation. Of course, if the patient is over 65 , or is feeble and not likely to live long in any case, we may then rightly choose the short-circuiting operation rather than the radical removal. The nature and scope of the operation and the.anastomosis which should be performed when removing a carcinoma of the colon depend naturally upon the situation and extent of the growth, and the same operation will be done whether it is being performed as a primary removal of a non-obstructed growth or as a secondary operation after acute obstruction has been relieved a week or two before. The procedure in general consists in mobilizing and freeing the growth and that part of the colon in which it lies, if necessary, and then removing the growth, a certain amount of bowel above and below it, and a deep V-shaped piece of the mesentery right down to the mesenteric root, and calculated to include all the glands and lymphatic vessels which drain that portion of the colon to be removed, all these structures being excised in one piece. As these growths appear to extend microscopically rather further up the bowel than down it; and to spread especially in the submucous layer, it is customary to remove rather more bowel above the growth than below it. The ends of the bowel are then joined together, either by lateral, end-to-end, or end-toside anastomosis, and it is a wise precaution to prevent an undue distension of the bowel above the junction with gas by making a temporary caecostomy as a safety valve. If the union is low down in the colon this object will be equally well achieved by stretching the anal sphincter, or by passing a flatus tube up from the anus through the anastomosis and leaving it in place for a few days.

So constant are these colonic growths in position that the operations which are performed for their removal are now more or less standardized. Thus for growths in the caecum or right-hand side of the colon it is necessary to remove the whole of both the caecum and the ascending colon in order to be certain of keeping outside the limits of the growth, and as by doing this we are liable to interfere with the blood supply of the lowest few inches of the ileum, and also to leave a blind pocket of transverse colon near the hepatic flexure, it has become customary in cases of carcinoma in this situation to remove the lowest five inches of the ileum, the caecum, the ascending colon, and a few inches of the transverse colon, and then to re-establish the continuity of the gut by uniting the ileum to the transverse colon by either side-to-side or end-to-end union. In this case there is no means of providing a safety valve above the anastomosis, nor is there any need so to do, as the faeces are here quite liquid. This operation must, of course, be preceded by mobilizing the caecum and ascending colon by means of an incision through the peritoneum of the posterior abdominal wall, on the outer side of the colon, and it will also entail a dissection of the lymphatics and glands up to the origin of the superior mesenteric artery. In the case of growths of the transverse and pelvic colons, where there is a well marked mesentery, simple resection of the growth with a deep $\mathrm{V}$ of the mesentery and end-toend union is sufficient; while if the carcinoma is in the descending colon or splenic flexure, as before, mobilization of the flexure and colon will be necessary, and then we must remove a few inches of the transverse colon, the flexure, and the whole of the descending colon. When dealing with portions of the large bowel which are not completely covered with peritoneum, such as the descending colon, it is well to remember that it is not wise to perform end-to-end anastomosis, and that lateral or end-to-side unions are preferable if possible. When performing a lateral or side-to-side anastomosis on the left of the colon; as after removal of the splenic flexure, I usually deliberately make an antiperistaltic anastomosis instead of the usual isoperistaltic one, for this reproduces to a certain extent the natural angle of the splenic flexure and turns the closed ends of the bowel and the raw edges where the mesentery has been cut, out into the retroperitoneal tissues and prevents them forming intraperitoneal adhesions.

Some knowledge of the relative degrees of malignancy of the three types of growth which are met with, and to which reference has been made above, is important; for in the most malignant type-the hard, craggy ulcer-one would feel justified in performing a more extensive and more radical operation than would be necessary for one of the other two less malignant varieties, and we can, during the operation, balance in our mind the extent of the procedure rendered necessary by the degree of malignancy of the particular type of growth present, and compare it with the patient's strength and ability to stand such a procedure.

The diagnosis of carcinoma of the colon in some ways resembles that of carcinoma of the stomach, for both are obvious in the later stages, but are difficult of diagnosis in the early stages, and this is the time when they should be subjected to operation if we are to offer the patient a reasonable chance of non-recurrence. Of both diseases the surgeon sees more cases which are inoperable than operable; this alone should be sufficient to make us feel that, on a reasonable suspicion that either of these diseases is present, an exploratory laparotomy is more than justifiable.

\section{THE CUTANEOUS TUBERCULIN TEST} applied to a Series of Definitely Diagnosed Caseg oF TUBERCULOSIS.

BY

S. L. CUMMINS, C.B., C.M.G., M.D.

DAVID DAVIES PROFESSOR OF TUBERCULOSIS, WELSH NATIONAL SCHOOR OF MEDICINE, CARDIFF, AND PRINCIPAL MEDICAL OFFICER, WELSH NATIONAL MEMORIAL ASSOCIATION.

In the British Medical Journal of April 21st, 1923 (p. 673), Dr. Charles McNeil describes a method of carrying out the cutaneous tuberculin test as follows:

"A small circular area of skin on the front of the forearm is chafed with the sharp point of a darning-needle until the epidermis is removed and the pink cutis vera exposed. Care is taken to avoid bleeding. The eye of the needle is now charged with undiluted old tuberculin, and pressed into the prepared vascular surface with a rotary motion. A small bruised pit is thus formed from which the tuberculin is rapidly absorbed."

In the course of an investigation into a series of cases of tuberculosis, some pulmonary, others surgical, under treatment in certain of the residential institutions of the Welsh 